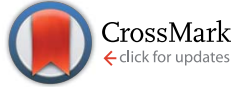

Cite this: J. Mater. Chem. A, 2016, 4, 14703

Received 7th June 2016

Accepted 24th August 2016

DOI: $10.1039 / c 6 t a 04770 j$

www.rsc.org/MaterialsA

\section{n-Doping of organic semiconductors for enhanced electron extraction from solution processed solar cells using alkali metals}

\author{
Tanja Schneider, ${ }^{a}$ Jens Czolk, ${ }^{a}$ Dominik Landerer, ${ }^{a}$ Stefan Gärtner, ${ }^{a}$ Andreas Puetz, ${ }^{a}$ \\ Michael Bruns, ${ }^{b}$ Jan Behrends ${ }^{c}$ and Alexander Colsmann*a
}

\begin{abstract}
To improve charge carrier injection into or extraction from organic optoelectronic devices, electrically doped layers are often employed. Whereas $n$-doping of organic semiconductors has been widely used in vacuum processed optoelectronic devices, adequate solution processes to enable future device printing are underdeveloped. In this work, we study n-doping of 1,3,5-tris(1-phenyl-1H-benzimidazol-2-yl) benzene (TPBi) in a solution process, using sodium as the electron donor. Upon addition of elementary sodium to a clear TPBi/toluene solution, we observed a change in color, indicating charge carrier transfer between sodium and TPBi. The optical and electrical properties of doped and undoped TPBi were characterized in solution and in the corresponding thin-films. Electron Paramagnetic Resonance (EPR) measurements revealed an increase of the number of unpaired spins upon doping, indicating the presence of doping-induced charge carriers. Implementing TPBi : Na as electron extraction layers in organic solar cells, we found almost the same device performance as compared to state-of-the-art solar cells comprising zinc oxide electron extraction layers.
\end{abstract}

\section{Introduction}

Common organic solar cells (OSCs) and organic light emitting diodes (OLEDs) comprise photo-active layers sandwiched between two electrodes. To ensure ohmic contacts between the electrodes and the photo-active layers and hence to improve charge carrier injection or extraction, electrical doping of organic interlayers has been extensively studied in vacuum processed organic devices. ${ }^{1-3}$ For p-doping, strong acceptors such as 2,3,5,6-tetrafluoro-7,7,8,8-tetracyanoquinodimethane $\left(\mathrm{F}_{4} \mathrm{TCNQ}\right)$ or $1,4,5,8,9,12$-hexaazatriphenylene $\left(\mathrm{HATCN}_{6}\right)$ have been employed to deplete electrons on the highest molecular orbital (HOMO) of the matrix molecule, generating hole excess. $^{4,5}$ Upon n-doping, an electron is transferred from a dopant to the lowest unoccupied molecular orbital (LUMO) of the matrix molecule. Common n-dopants are cationic dyes $^{6-8}$ or alkali metals with low ionization energies. ${ }^{\mathbf{9 1 0}}$ For example, Kido et al. reported that, upon doping of evaporated aluminium-tris(8-hydroxychinolin) $\left(\mathrm{Alq}_{3}\right)$ with lithium (Li), radicals are formed, i.e., electrons are transferred from $\mathrm{Li}$ to

${ }^{a}$ Light Technology Institute, Karlsruhe Institute of Technology (KIT), Engesserstrasse 13, 76131 Karlsruhe, Germany. E-mail: alexander.colsmann@kit.edu

${ }^{b}$ Institute for Applied Materials and Karlsruhe Nano Micro Facility (KNMF), Karlsruhe Institute of Technology (KIT), Hermann-von-Helmholtz-Platz 1, 76344 EggensteinLeopoldshafen, Germany

${ }^{c}$ Berlin Joint EPR Lab, Fachbereich Physik, Freie Universität Berlin, Arnimallee 14, 14195 Berlin, Germany
$\mathrm{Alq}_{3} \cdot{ }^{11}$ Moses et al. demonstrated n-doping of polyacetylene with alkali metals by exposing the polymer film to alkali vapor. ${ }^{\mathbf{1 2}}$ However, this process is not suitable for industrial device fabrication.

In light of the prospect of employing printing and coating techniques for future large-area roll-to-roll device fabrication, electrical doping of organic layers will have to be achieved through solution processing. ${ }^{13}$ Whereas p-doping of molecular materials and polymers from solution has been reported in the literature before, ${ }^{\mathbf{1 4}} \mathrm{n}$-doping of organic molecules using solution-based processes appears more challenging due to the non-solubility of alkali metals in most processing agents. Rare examples of $\mathrm{n}$-doping from solution utilize organometallic dimers or dimers of benzimidazoline radicals. ${ }^{15,16}$ Alkali metals are soluble in ammonia at a process temperature of $-78{ }^{\circ} \mathrm{C}$, not being suitable for any relevant deposition processes.

In this work, we dissolved sodium in 1,3,5-tris(1-phenyl-1Hbenzimidazol-2-yl)benzene (TPBi)/toluene solutions at room temperature, enabling the solution deposition of n-doped organic layers. We investigated the n-doped and undoped TPBi solutions by UV-Vis absorption spectroscopy and studied the electrical properties of the respective TPBi : Na bulks by Electron Paramagnetic Resonance (EPR) and conductivity measurements. The n-doped layers were then incorporated into organic solar cells, replacing the widely employed zinc oxide (ZnO) electron extraction layers. 


\section{Experimental}

Toluene (Sigma-Aldrich, anhydrous, 99.8\%) was dried over sodium (Na) to remove water and oxygen. The dopant sodium (Chempur, in oil, 99.95\%) was cut under inert atmosphere and cleaned from residual mineral oil. TPBi (Syntec-Sensient, 99.95\%) was dissolved (10 $\left.\mathrm{g} \mathrm{L}^{-1}\right)$ in dry toluene, and the dopant sodium was added to the TPBi/toluene solution. After stirring the solution overnight, the solid sodium surplus was removed.

Absorbance spectra of the solutions were measured using a Lambda 1050 UV-Vis-NIR spectrophotometer (Perkin Elmer).

Nuclear magnetic resonance (NMR) spectra were measured by a Bruker Avance III Microbay $400 \mathrm{MHz}$. Therefore, tolueneD8 solutions of TPBi : Na or, for reference, neat TPBi were filled into NMR tubes under inert atmosphere and sealed using a blowtorch.

Room temperature continuous wave electron paramagnetic resonance (cwEPR) spectra were recorded on a laboratory-built $\mathrm{X}$-band EPR spectrometer to detect unpaired spins in TPBi : Na films. The respective solutions were filled in EPR tubes, the solvent was removed in vacuo, the tubes were filled with inert gas and sealed using a blowtorch. To control the magnetic field, a field controller (Bruker BH15) was used. Microwaves were generated and monitored by a microwave bridge (Bruker ER 048 R). Magnetic-field modulation in combination with lock-in detection was employed using a lock-in amplifier (Stanford Research SR810) and a modulation amplifier (Wangine WPA-120), resulting in derivative spectra. To determine the quality factor $Q$ of the sample in a Bruker ER 4122 SHQ microwave resonator, a mode picture was measured before each measurement started. The measured EPR signal was double integrated and compared to a 4-hydroxy-TEMPO-reference sample with a known number of spins. This comparison yielded the absolute number of spins. ${ }^{17}$ The uncertainty with respect to the absolute number of unpaired spins is less than $20 \%$, which is estimated based on the uncertainty of the sample volume when filling the tubes.
Thin-film conductivities $\sigma$ were measured in electron-only devices comprising a spin cast $30 \mathrm{~nm}$ thick undoped TPBi or doped TPBi : Na layer, respectively, sandwiched between two thermally evaporated aluminum electrodes. The conductivities were calculated from the linear regime of the current densityvoltage $(J-V)$ curves at $0.04 \mathrm{~V}$ using Ohm's law.

Amplitude Modulation Kelvin Probe Force Microscopy (AMKPFM) dual pass experiments were carried out in tapping mode on a Bruker Dimension ICON using a Pt/Ir coated FMV-PT tip to measure the contact potential difference $\mathrm{CPD}=-\left(\Phi_{\text {tip }}-\right.$ $\left.\Phi_{\text {sample }}\right) / \mathrm{e}, \Phi<0$. The CPD was referenced with the work function of highly oriented pyrolytic graphite $\Phi_{\mathrm{HOPG}}=-4.4 \mathrm{eV} \cdot{ }^{18}$

Solar cells were built on ITO coated glass substrates $\left(R_{\square}=13\right.$ $\Omega \mathrm{sq}^{-1}$ ) according to the device architecture depicted in Fig. 1a. The substrates were successively cleaned in acetone and 2-propanol in an ultrasonic bath and treated with oxygen plasma. Afterwards, the samples were transferred into a glovebox and kept under nitrogen atmosphere for the remaining fabrication and characterization process. Electron extraction layers (EELs) from either undoped or sodium doped TPBi (Fig. 1b) or, for reference, $\mathrm{ZnO}$ were employed. Therefore, $30 \mathrm{~nm}$ thick films of sodium doped or undoped TPBi were spin cast from toluene solution ( $10 \mathrm{~g} \mathrm{~L}^{-1}, 1000 \mathrm{rpm}, 30 \mathrm{~s}, 4000 \mathrm{rpm}, 3 \mathrm{~s}$ ). For reference, $30 \mathrm{~nm}$ thick films of $\mathrm{ZnO}$ were spin cast from nanoparticle dispersion (Nanograde N-10, Nanograde Ltd., $1 \mathrm{wt} \%$ in isopropanol, $4000 \mathrm{rpm}, 30 \mathrm{~s}$ ) and thermally annealed on a hotplate $\left(85{ }^{\circ} \mathrm{C}, 10 \mathrm{~min}\right)$. Poly[[2,6'-4,8-di(5-ethylhexylthienyl)benzo[1,2- $b ; 3,3-b]$ dithiophene][3-fluoro-2[(2-ethylhexyl)carbonyl]thieno[3,4- $b]$ thiophenediyl]] (PTB7-Th, 1-Material Inc., $M_{\mathrm{w}}=105 \mathrm{kDa}, \emptyset_{\mathrm{M}}=2$, Fig. 1c) and [6,6]-phenyl $\mathrm{C}_{61}$-butyric acid methyl ester ( $\mathrm{PC}_{61} \mathrm{BM}$, Solenne, 99\%, Fig. 1c) were dissolved $\left(1: 1.5, \mathrm{w}: \mathrm{w}, 23 \mathrm{~g} \mathrm{~L}^{-1}\right)$ in $o$-xylene (SigmaAldrich, anhydrous, 97\%) and stirred overnight at $85{ }^{\circ} \mathrm{C}$. The warm solution was spin cast ( $90 \mathrm{~nm}, 1500 \mathrm{rpm}, 60 \mathrm{~s}$ ) on top of the electron extraction layer. The molybdenum oxide/silver counter electrode $\left(\mathrm{MoO}_{x} / \mathrm{Ag}, 10 \mathrm{~nm} / 100 \mathrm{~nm}\right)$ was thermally evaporated through a shadow mask in an evaporation chamber

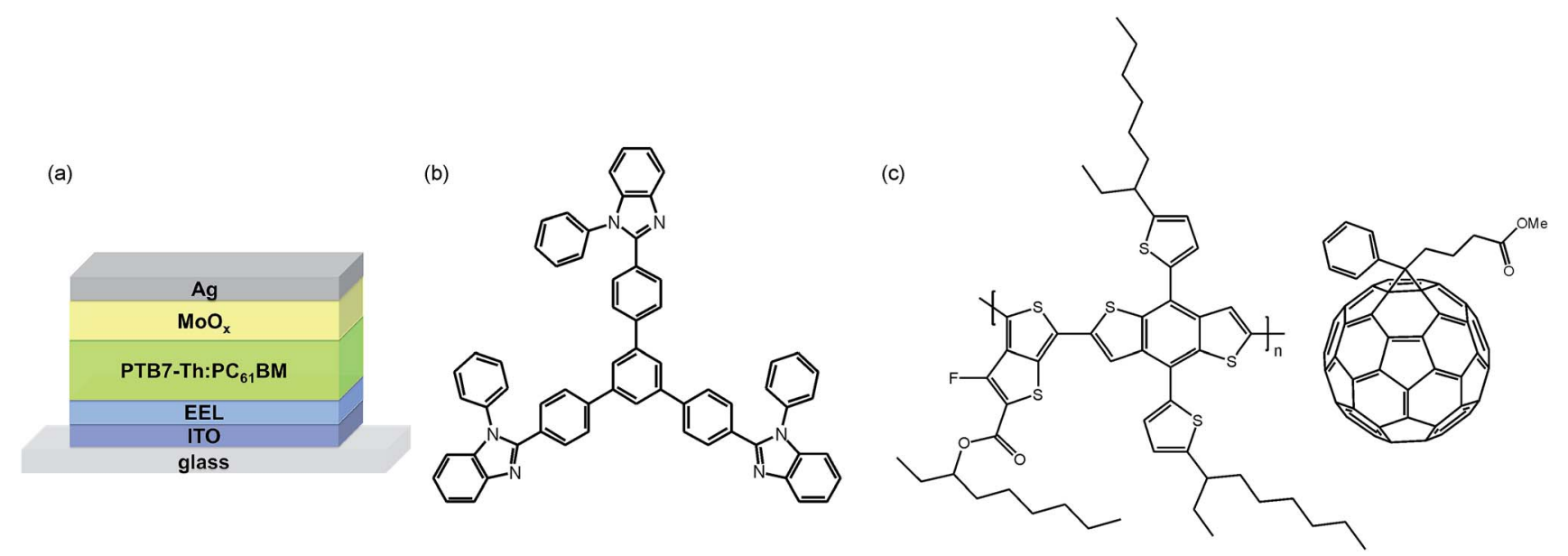

Fig. 1 (a) Device architecture of the PTB7-Th:PC ${ }_{61} B M$ solar cells comprising different electron extraction layers (EELs). Chemical structures of (b) TPBi and (c) the bulk-heterojunction components PTB7-Th and $\mathrm{PC}_{61} \mathrm{BM}$. 
$\left(10^{-6} \mathrm{mbar}\right)$ attached to the glovebox, defining the photo-active area of the solar cell $\left(3 \times 3.5 \mathrm{~mm}^{2}\right)$.

Current density-voltage curves were recorded with a source measure unit (Keithley 238) under illumination from a spectrally monitored solar simulator (Oriel $300 \mathrm{~W}, 1000 \mathrm{~W} \mathrm{~m}^{-2}$, ASTM AM 1.5G), calibrated by a KG5 filtered silicon reference cell (91150-KG5, Newport). The sample edges were masked to avoid light-incoupling through substrate modes.

Film thicknesses were measured by a tactile stylus surface profiler (Bruker Dektak XT).

Time-of-flight secondary ion mass spectrometry was performed on a TOF-SIMS ${ }^{5}$ instrument (ION-TOF GmbH, Münster, Germany), equipped with a Bi cluster liquid metal primary ion source and a non-linear time-of-flight analyser. The Bi source was operated in "bunched" mode providing $1 \mathrm{~ns} \mathrm{Bi}_{3}{ }^{+}$ion pulses at $25 \mathrm{keV}$ energy, an analysed area of $100 \times 100 \mu \mathrm{m}^{2}$ and a lateral resolution of approx. $4 \mu \mathrm{m}$. Negative polarity spectra were calibrated on the $\mathrm{C}^{-}, \mathrm{C}_{2}{ }^{-}, \mathrm{C}_{3}{ }^{-}$, and $\mathrm{C}_{4}{ }^{-}$peaks. Positive polarity spectra were calibrated on the $\mathrm{C}^{+}, \mathrm{CH}^{+}, \mathrm{CH}_{2}{ }^{+}$, and $\mathrm{CH}_{3}{ }^{+}$ peaks. Sputter depth profiles were performed using a $1 \mathrm{keV} \mathrm{Cs}^{+}$ ion beam and a raster size of $500 \times 500 \mu \mathrm{m}^{2}$.

\section{Results and discussion}

In order to avoid losses in the open-circuit voltage $\left(V_{\text {oc }}\right)$ of organic solar cells, as a rule of thumb, EELs with Fermi energies higher than or equal to the electron affinity of the fullerene acceptor have to be chosen. TPBi complies with this requirement, having a LUMO energy of about $-2.8 \mathrm{eV}^{\mathbf{1 9}}$ and, upon ndoping, a fermi level energy close to the LUMO energy. With an ionization energy of about $2.75 \mathrm{eV},{ }^{20} 3 \mathrm{~s}$ valence electrons of sodium can transfer to TPBi, forming electron excess on the TPBi matrix. Whereas alkali metal doping is widely used in vacuum deposited organic semiconductor devices, its applicability by wet processing is widely unexplored, since neat alkali metals do not dissolve in common processing agents. However, when adding a piece of sodium to a $10 \mathrm{~g} \mathrm{~L}^{-1}$ solution of TPBi in toluene, the clear TPBi/toluene solution turned red (Fig. 2, inset). The respective UV-Vis absorbance spectrum depicted in Fig. 2 shows additional absorption bands that hint towards new polaronic states in the reduced TPBi. ${ }^{21}$ Notably, the reduction of the TPBi molecules is reversible: after exposing the $\mathrm{TPBi}^{-} / \mathrm{Na}^{+} /$ toluene solution to air, the solution turns clear again.

To explore changes of the molecular conformation after reducing the TPBi molecules, we recorded NMR spectra of the TPBi/toluene and the $\mathrm{TPBi}^{-} / \mathrm{Na}^{+} /$toluene solution as shown in Fig. 3. Upon doping, we observed a broadening of all peaks, which can be attributed to an oligomerization of the TPBi molecules, ${ }^{22}$ initiated by the formation of radicals after electron transfer from sodium to TPBi. We note that the oligomerization of TPBi in solution does not affect the principle electron excess on the TPBi molecules.

After the analysis of the $\mathrm{TPBi}^{-} / \mathrm{Na}^{+} /$toluene solution, we investigated the effect of doping on the solid TPBi : Na bulk and, for reference, neat TPBi samples. Therefore we filled $50 \mu \mathrm{l}$ of the $\mathrm{TPBi}^{-} / \mathrm{Na}^{+} /$toluene or TPBi/toluene solution in EPR tubes, removed the solvent under vacuum and sealed the tubes. In

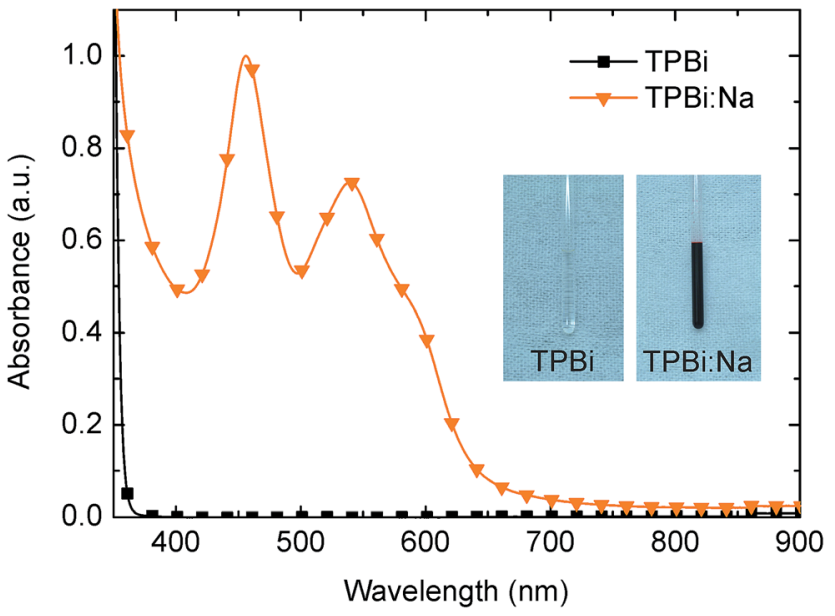

Fig. 2 Normalized UV-Vis absorbance spectra of neat TPBi/toluene and $\mathrm{TPBi}^{-} / \mathrm{Na}^{+} /$toluene solutions. Upon addition of sodium, absorption bands between 350 and $650 \mathrm{~nm}$ occur, indicating the formation of new polaronic states through transfer of the 3 s electrons from sodium to TPBi.

order to investigate if the reduced $\mathrm{TPBi}^{-}$molecules prevailed, we measured the number of unpaired electrons within the dry thin-films by EPR. The Zeeman interaction between the magnetic momentums associated with unpaired electron spins and an external magnetic field causes a splitting between two states with different magnetic spin quantum number $\left(m_{\mathrm{s}}= \pm \frac{1}{2}\right)$. Transitions between the two energy levels can be induced by resonant microwave absorption. In typical cwEPR experiments, field modulation in combination with lock-in detection is used, which leads to the typical derivative EPR line shape. The quality factor $Q$ of the resonator loaded with the sample was determined from the mode picture before each EPR

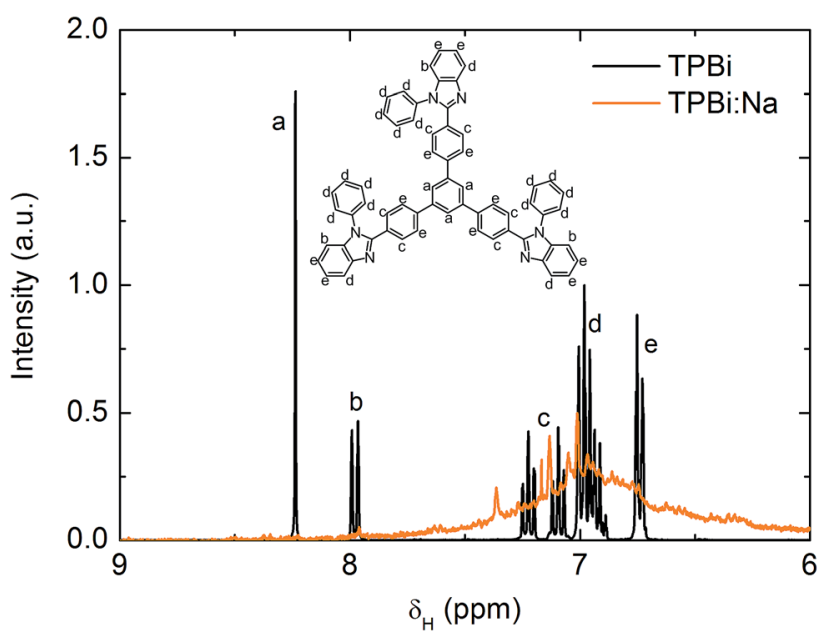

Fig. 3 NMR spectra of neat TPBi and TPBi : Na solutions in tolueneD8. The spectrum of undoped TPBi exhibits sharp peaks whereas the peaks of the doped TPBi are much broader. We attribute this broadening to an oligomerization of TPBi molecules induced by radical formation due to the electron transfer from sodium to TPBi. For better visibility, the spectra have been plotted using arbitrary units. 
measurement was started. The measured derivative spectra were background-corrected and double-integrated to obtain the integrated EPR signal amplitude. As depicted in Fig. 4, layers of undoped TPBi show weak background signatures of unpaired electrons. However, for thin-films deposited from $\mathrm{TPBi}^{-} / \mathrm{Na}^{+} /$ toluene solution, a significantly stronger EPR signal can be detected, indicating the presence of doping-induced electrons in the TPBi layer. The absolute number of spins per sample was calculated by double-integration of the EPR signal and comparison to a 4-hydroxy-TEMPO-reference sample with a known number of spins. Spin concentrations were calculated by dividing the total amount of measured spins by the sample volume, and the doping efficiencies were determined by neglecting the number of sodium atoms and considering the fraction of $n$-doped TPBi molecules. The concentration of unpaired spins increases from $1 \times 10^{16} \mathrm{~cm}^{-3}$ for undoped TPBi by more than one order of magnitude to $4 \times 10^{17} \mathrm{~cm}^{-3}$ for TPBi : Na. This spin concentration corresponds to 1 unpaired electron per 5000 TPBi molecules. The actual number of electrons transferred from $\mathrm{Na}$ to TPBi is higher, with the electrons from TPBi radicals that were oligomerized, not being visible in EPR measurements.

To investigate the effect of Na-doping on the physical properties of TPBi thin-films, we sandwiched $30 \mathrm{~nm}$ thick films between two aluminum electrodes and measured their $J-V$ characteristics. The films were spin cast from either the TPBi or the $\mathrm{TPBi}^{-} / \mathrm{Na}^{+}$solutions. From the $J-V$ curves at $0.04 \mathrm{~V}$, we estimated the conductivity of undoped films on the order of $10^{-9} \mathrm{~S} \mathrm{~cm}^{-1}$ whereas the conductivity improved by two orders of magnitude to $10^{-7} \mathrm{~S} \mathrm{~cm}^{-1}$ in doped films.

Ideally, n-doping of TPBi does not only enhance the conductivity of films but also improves the extraction (solar cells) or injection (OLEDs) of electrons. Therefore, we probed the work functions of TPBi and TPBi : Na thin-films by KPFM. Upon Na-doping, the work function shifted from $-4.40 \mathrm{eV}$

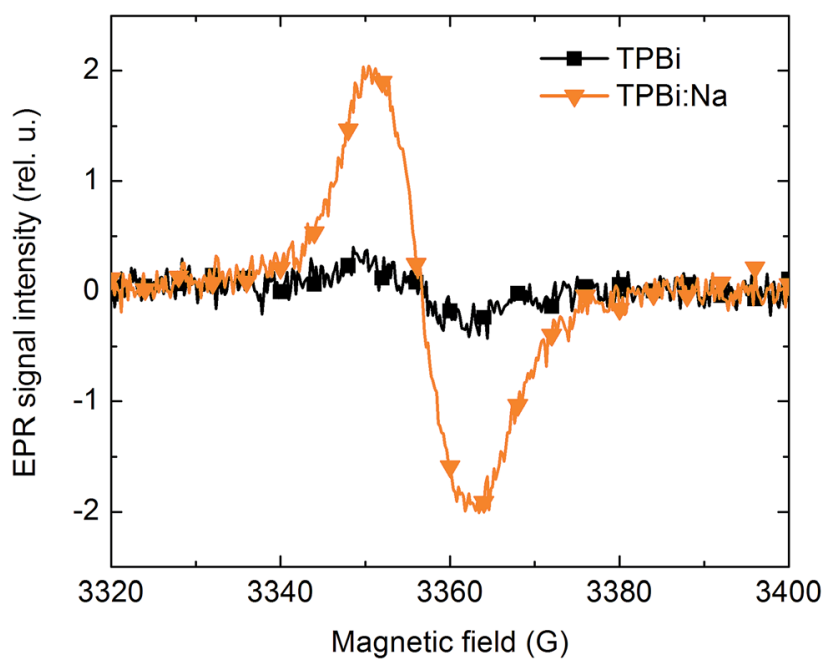

Fig. 4 EPR spectra of neat TPBi and TPBi: Na layers. Whereas undoped TPBi exhibits only weak EPR signals, TPBi : Na layers show a strong signature of unpaired spins which can be attributed to doping-induced electrons and hence to $n$-doping of the TPBi. (undoped TPBi) to $-3.55 \mathrm{eV}$, indicating a Fermi level shift towards the TPBi LUMO. As a consequence, in solar cells, photogenerated electrons on $\mathrm{PC}_{61} \mathrm{BM}$ face a substantial extraction barrier towards neat TPBi, hampering the extraction of charges. Likewise, in OLEDs, undoped TPBi will form a high injection barrier with the cathode. Upon shifting the Fermi level towards the TPBi LUMO, both barriers are reduced, enabling efficient charge carrier extraction or injection, respectively.

We exemplify the merits from solution processed TPBi : Na EELs on organic solar cells featuring a glass/ITO/EEL/PTB7$\mathrm{Th}: \mathrm{PC}_{61} \mathrm{BM} / \mathrm{MoO}_{x} / \mathrm{Ag}$ device architecture as depicted in Fig. 1a, employing a $30 \mathrm{~nm}$ EEL from either neat (undoped) TPBi, sodium doped (n-doped) TPBi or, for reference, nanoparticulate $\mathrm{ZnO}$ that allows for comparison with the literature. The $90 \mathrm{~nm}$ thick PTB7-Th:PC ${ }_{61} \mathrm{BM}$ photo-active layer was deposited from $o$ xylene, intentionally omitting processing additives such as 1,8diiodooctane or $p$-anisaldehyde that would chemically react with the sodium in the underlying EEL.

The integrity of the layer stacks and in particular the presence of an intact TPBi : Na EEL was probed by ToF-SIMS on the same devices used for $J-V$ measurements. To simultaneously investigate sodium diffusion within the solar cells, we deliberately stored the devices for six months after fabrication and $J-V$ characterization. During ToF-SIMS measurements, the layers were successively ablated with a $\mathrm{Cs}^{+}$ion beam while positively and negatively charged ions were detected. The ToF-SIMS signal and the corresponding layers of the solar cell are depicted in Fig. 5. Starting the analysis from the $\mathrm{Ag}$ top electrode $\left(\mathrm{CsAg}^{+}\right)$, we sequentially identified the $\mathrm{MoO}_{x}$ layer $\left(\mathrm{CsMo}^{+}\right)$, the photo-active PTB7-Th:PC ${ }_{61} \mathrm{BM}$ layer $\left(\mathrm{CS}^{-}\right)$, the TPBi : Na EEL $\left(\mathrm{C}_{2} \mathrm{~N}_{2}{ }^{-}\right)$and the ITO bottom electrode $\left(\mathrm{In}_{2} \mathrm{O}_{2}{ }^{-}\right)$. The $\mathrm{CsNa}^{+}$signal provided information about the sodium content throughout the layer

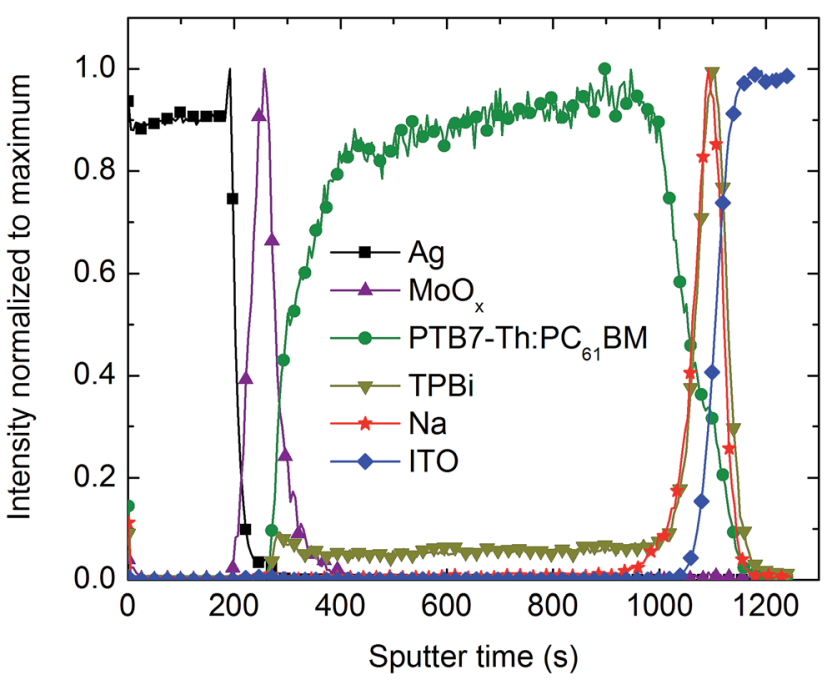

Fig. 5 ToF-SIMS measurements on a typical solar cell comprising a TPBi : $\mathrm{Na}$ layer. $\mathrm{Ag}\left(\mathrm{CsAg}^{+}\right), \mathrm{MoO}_{x}\left(\mathrm{CsMo}^{+}\right)$and $\mathrm{Na}\left(\mathrm{CsNa}{ }^{+}\right)$were detected in the positive polarity spectrum. PTB7-Th:PC $\left.{ }_{61} \mathrm{BM}_{(\mathrm{CS}}^{-}\right)$, TPBi $\left(\mathrm{C}_{2} \mathrm{~N}_{2}^{-}\right)$and ITO $\left(\mathrm{In}_{2} \mathrm{O}_{2}^{-}\right)$were detected in the negative polarity spectrum. The co-location of TPBi and Na proves the integrity of the TPBi : Na electron extraction layer between the photo-active PTB7Th: $\mathrm{PC}_{61} \mathrm{BM}$ layer and the ITO cathode. 
stack. For better readability, all curves were normalized to their maxima, leading to a somewhat higher (artificial) background noise of the $\mathrm{C}_{2} \mathrm{~N}_{2}{ }^{-}$signal. Not only did we find the sodium peak coinciding with the TPBi layer, indicating an intact TPBi : Na EEL. We also found that sodium did not diffuse within the device, which is an often observed process that is detrimental to the device performance. ${ }^{23}$ We carried out the same measurement for reference devices comprising undoped TPBi layers and found the TPBi layer preserved at its location in the layer stack, too (data not shown here).

Even without additives, the reference PTB7-Th:PC ${ }_{61} \mathrm{BM}$ cells with ZnO EELs yielded hero efficiencies of almost 7\%, thereby outperforming additive-free, literature-known solar cells. ${ }^{24,25}$ $\mathrm{ZnO}$ is an efficient EEL with a work function $\Phi_{\mathrm{ZnO}}=4.1 \mathrm{eV}$ which is often employed for electron extraction in organic solar cells due to its excellent electronic interface formation with $\mathrm{PC}_{61} \mathrm{BM}$. With $\Phi_{\mathrm{ZnO}}=4.1 \mathrm{eV}$ being close to the LUMO of $\mathrm{PC}_{61} \mathrm{BM}$, typically, the $V_{\mathrm{oc}}=812 \mathrm{mV}$ does not suffer any losses but rather achieves the maximum possible value. Fig. 6 depicts the corresponding current density-voltage curves. Their key performance data open-circuit voltage $\left(V_{\mathrm{oc}}\right)$, short-circuit current density $\left(J_{\mathrm{sc}}\right)$, fill factor $(\mathrm{FF})$ and power conversion efficiency (PCE) are summarized in Table 1.

Under reverse bias and hence upon field-assisted charge carrier extraction, all solar cells exhibit the same saturation current density, which indicates about equal photo-generation of charge carriers in all three devices. However, under shortcircuit conditions, the photo current of the solar cell comprising an undoped TPBi layer is reduced as TPBi forms a barrier for electrons that hampers their collection by the cathode and negatively affects the $\mathrm{FF}=28 \%$. At the same time, the reduced built-in field affects the $V_{\mathrm{oc}}=290 \mathrm{mV}$. Altogether, the solar cells with TPBi EEL yield a PCE $=0.7 \%$ only. Upon n-doping of TPBi with sodium, the extraction barrier is effectively reduced almost

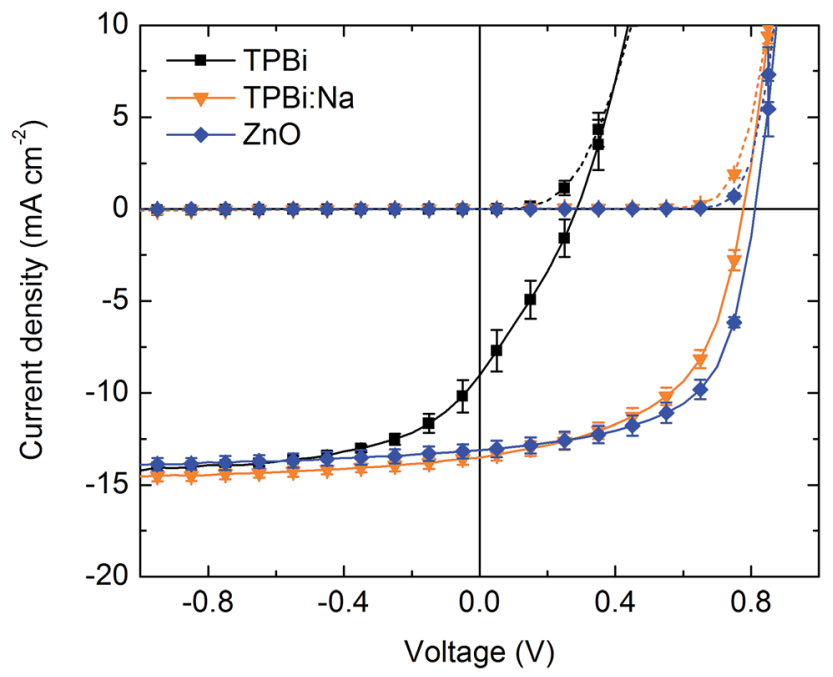

Fig. 6 Typical J-V curves of glass/ITO/EEL/PTB7-Th:PC ${ }_{61} \mathrm{BM} / \mathrm{MoO}_{x} /$ Ag solar cells under AM1.5 illumination. The solar cell with a TPBi : Na EEL exhibits a higher $V_{\text {oc }}$ and FF than the solar cell comprising neat TPBi and almost matches the performance of the reference solar cell with an EEL from $\mathrm{ZnO}$.
Table 1 Key performance data of organic solar cells comprising different electron extraction layers (EELs), averaged over 6-12 cells. Performances of hero devices in parentheses

\begin{tabular}{llrrl}
\hline & $V_{\text {oc }}(\mathrm{mV})$ & $J_{\text {sc }}\left(\mathrm{mA} \mathrm{cm}^{-2}\right)$ & FF $(\%)$ & PCE $(\%)$ \\
\hline TPBi & $290 \pm 20$ & $9.0 \pm 1.1$ & $28 \pm 1$ & $0.7 \pm 0.2(0.9)$ \\
TPBi $:$ Na & $777 \pm 5$ & $13.5 \pm 0.3$ & $54 \pm 1$ & $5.6 \pm 0.3(6.1)$ \\
ZnO & $812 \pm 6$ & $13.1 \pm 0.4$ & $60 \pm 2$ & $6.4 \pm 0.3(6.8)$
\end{tabular}

reinstalling the full $V_{\mathrm{oc}}=777 \mathrm{mV}$ and a $\mathrm{FF}=54 \%$, yielding a hero PCE $=6.1 \%$ which is only $10 \%$ (rel.) below the performance of the $\mathrm{ZnO}$ reference device.

We note that, in preliminary experiments, UV-Vis absorption measurements and solar cell device data hinted towards similar physical properties of other semiconductor/alkali metal combinations, such as potassium doped 2-(4-tert-butylphenyl)5-(4-biphenylyl)-1,3,4-oxadiazole (BuPBD : K) or lithium doped bathophenanthroline (BPhen : Li). This indicates that doping of organic semiconductors with alkali metals from solution is a more general concept that can be adopted for a variety of material combinations.

\section{Conclusions}

n-Doping can be achieved employing wet processes, when solid alkali metals (here: sodium) are added to an organic semiconductor solution. The electrical doping effect endures the thin-film deposition processes under inert atmosphere. They can be incorporated into organic solar cells where they enhance electron extraction. In contrast to $\mathrm{ZnO}$, the doped organic layers do not require thermal annealing. Beyond solar cells, this n-doping process may be a versatile asset of the organic semiconductor toolbox for future low-temperature solution fabrication of other organic optoelectronic devices such as OLED or thermoelectric generators.

\section{Acknowledgements}

We thank Silke Wolf for NMR measurements, Daniel Bahro for fruitful discussions and Nanograde for supplying $\mathrm{ZnO}$ nanoparticle dispersions. This work was funded by the Federal Ministry of Education and Research under contract no. 03EK3504 (project TAURUS) and the German Research Council (DFG) within SFB 1176 in the context of project "Highly crosslinked polymers for functional multi-layers" and within the SPP 1601 in the context of project "Transient EDMR". T. S. acknowledges the Karlsruhe School of Optics \& Photonics (KSOP) for support.

\section{Notes and references}

1 K. Walzer, B. Maennig, M. Pfeiffer and K. Leo, Chem. Rev., 2007, 107, 1233-1271.

2 B. Lüssem, M. Riede and K. Leo, Phys. Status Solidi A, 2013, 210, 9-43. 
3 M. Pfeiffer, K. Leo, X. Zhou, J. S. Huang, M. Hofmann, A. Werner and J. Blochwitz-Nimoth, Org. Electron., 2003, 4, 89-103.

4 J. Blochwitz, M. Pfeiffer, M. Hofmann and K. Leo, Synth. Met., 2002, 127, 169-173.

5 Y.-K. Kim, J. Won Kim and Y. Park, Appl. Phys. Lett., 2009, 94, 063305.

6 F. Li, M. Pfeiffer, A. Werner, K. Harada, K. Leo, N. Hayashi, K. Seki, X. Liu and X.-D. Dang, J. Appl. Phys., 2006, 100, 23716.

7 A. G. Werner, F. Li, K. Harada, M. Pfeiffer, T. Fritz and K. Leo, Appl. Phys. Lett., 2003, 82, 4495-4497.

8 F. Li, A. Werner, M. Pfeiffer, K. Leo and X. Liu, J. Phys. Chem. $B$, 2004, 108, 17076-17082.

9 J. Huang, M. Pfeiffer, A. Werner, J. Blochwitz, K. Leo and S. Liu, Appl. Phys. Lett., 2002, 80, 139-141.

10 T. Uchida, S. Kaneta, M. Ichihara, M. Ohtsuka, T. Otomo and D. R. Marx, Jpn. J. Appl. Phys., 2005, 44, L282-L284.

11 J. Kido and T. Matsumoto, Appl. Phys. Lett., 1998, 73, 28662868.

12 D. Moses, N. Colaneri and A. J. Heeger, Solid State Commun., 1986, 58, 535-539.

13 F. Nickel, T. Haas, E. Wegner, D. Bahro, S. Salehin, O. Kraft, P. A. Gruber and A. Colsmann, Sol. Energy Mater. Sol. Cells, 2014, 130, 317-321.
14 S. Feser and K. Meerholz, Chem. Mater., 2011, 23, 5001-5005. 15 S. Guo, S. B. Kim, S. K. Mohapatra, Y. Qi, T. Sajoto, A. Kahn, S. R. Marder and S. Barlow, Adv. Mater., 2012, 24, 699-703.

16 B. D. Naab, S. Zhang, K. Vandewal, A. Salleo, S. Barlow, S. R. Marder and Z. Bao, Adv. Mater., 2014, 26, 4268-4272.

17 G. R. Eaton, S. S. Eaton, D. P. Barr and R. T. Weber, Quantitative EPR, Springer Vienna, Vienna, 2010.

18 H. Ago, T. Kugler, F. Cacialli, K. Petritsch, R. H. Friend, W. R. Salaneck, Y. Ono, T. Yamabe and K. Tanaka, Synth. Met., 1999, 103, 2494-2495.

19 S. H. Kim, J. Jang and J. Y. Lee, Appl. Phys. Lett., 2007, 91, 083511.

20 H. B. Michaelson, J. Appl. Phys., 1977, 48, 4729-4733.

21 S. Sivaramakrishnan, M. Zhou, A. C. Kumar, Z.-L. Chen, R.-Q. Png, L.-L. Chua and P. K. H. Ho, Appl. Phys. Lett., 2009, 95, 213303.

22 M. Hesse, H. Meier and B. Zeeh, Spectroscopic methods in organic chemistry, Thieme, Stuttgart, 2007.

23 G. Parthasarathy, C. Shen, A. Kahn and S. R. Forrest, J. Appl. Phys., 2001, 89, 4986-4992.

24 J. Kong, I.-W. Hwang and K. Lee, Adv. Mater., 2014, 26, 62756283.

25 C. Sprau, F. Buss, M. Wagner, D. Landerer, M. Koppitz, A. Schulz, D. Bahro, W. Schabel, P. Scharfer and A. Colsmann, Energy Environ. Sci., 2015, 8, 2744-2752. 